\title{
Exploitation of Cell Mediated Immune Responses to Cancer Immunotherapy
}

\author{
TILAYE ATNAF ASHINE ${ }^{1}$ TESFAYE SISAY TESEMA ${ }^{2}$ \\ 1.College of Natural and Computational Science Department of Biology (Stream of Medical Biotechnology), \\ Madda Walabu University, PO box 47, Bale Robe, Ethiopia \\ 2.Unit of Health Biotechnology, Director, Institute of Biotechnology, Addis Ababa University
}

\begin{abstract}
Individual human tumors arise through a combination of genetic and epigenetic changes that facilitate immortality. Cancer occurs when there are changes to genes that control the way our cells grow and divide.. The increase in the prevalence of cancer with lack of specific treatment options became public health problem particularly in middle and low income nations. Activation of the immune system by products of viral oncogene prevents viral induced cancers. The in vitro stimulation of Dendritic cells with tumor or tumor antigen in the presence of cytokines for cross activation of CD8+ and CD4+ $\mathrm{T}$ cells or restoration of $\mathrm{T}$ cells functions by blockade of immune blockers or modification tumor microenvironment or adoptive transfer of immune cells and stem $\mathrm{T}$ cells are promising cancer therapies. Compared with the convectional cancer therapies, immunotherapies are most tumors targeted without harmful side effects.
\end{abstract}

DOI: $10.7176 / \mathrm{JMPB} / 59-03$

Publication date:September $30^{\text {th }} 2019$

\section{Introduction}

Cancer is a cell that has lost its normal growth control mechanism. It is a disease of multicellular organism that results when the controls that regulate normal cell growth break down (Karp, 2013; Ponder, 2001). Cancers are generally caused by accumulation of mutations in in higher vertebrates. A tumor that is not capable of indefinite growth and does not invade the healthy surrounding tissue extensively is benign while a tumor that continues to grow and becomes progressively invasive is malignant. The term cancer refers specifically to a malignant tumor. Malignant tumors exhibit metastasis; i.e., in this process, small clusters of cancerous cells dislodge from a tumor, invade the blood or lymphatic vessels, and are carried to other tissues. In this way a primary tumor at one site can give rise to a secondary tumor at another site (Cooper and Hausman, 2007; Karp, 2013; Ponder, 2001) .

Cancer is a major health problem worldwide and one of the most important causes of morbidity and mortality in children and adults. Cancer has become the second-ranking cause of death in the Western world led only by heart disease. Approximately 14 million people across the world are diagnosed with cancer every year, about 8 million people die from this disease annually and more than 32.6 million people were living with cancer (Lacombe, 2016; Sanghi et. al., 2014; Yongshu et. al., 2016). About 57\% (approximately 8 million) of new arising cancer cases, 65\% (approximately 5.3 million) deaths of cancer and 48\% (approximately 15.6 million) of the 5-year prevalent cancer cases have taken place in underdeveloped areas (Yongshu et al., 2016). Approximately one out of every three Americans will develop cancer at some point in life and, in spite of major advances in treatment, nearly one out of every four Americans ultimately die of this disease. The lethality of malignant tumors is due to their uncontrolled growth within normal tissues, causing damage and functional impairment. The malignant phenotype of cancers reflects defects in regulation of cell proliferation, resistance of the tumor cells to apoptotic death, ability of the tumor cells to invade host tissues and metastasize to distant sites, and tumor evasion of host immune defense mechanisms (Ponder, 2001).

More than 100 kinds of human cancers have been identified (Sanghi et. al., 2014), and they are classified according to the embryonic origin of the tissue from which the tumor is derived. Most $(>80 \%)$ are carcinomas, tumors that arise from endodermal or ectodermal tissues such as skin or the epithelial lining of internal organs and glands. The majority of cancers of the colon, breast, prostate, and lung are carcinomas. The leukemias and lymphomas are malignant tumors of hematopoietic cells of the bone marrow and account for about $9 \%$ of cancer. Sarcomas, which arise less frequently, are derived from mesodermal connective tissues such as bone, fat, and cartilage (Karp, 2013).

The possibility that cancers can be eradicated by specific immune responses has been the main reason for a large body of work in the field of tumor immunology (Farkona et.al., 2016; Lacombe, 2016). The concept of immune surveillance of cancer, which was proposed in the 1950s, states that a physiologic function of the immune system is to recognize and destroy clones of transformed cells before they grow into tumors and to kill tumors after they are formed. Its existence has been demonstrated by the increased incidence of some types of tumors in immunocompromised experimental animals and humans. It is now clear that the innate and adaptive immune systems do react against many tumors, and exploiting these reactions to specifically destroy tumors is an important goal of tumor immunologists (Abbas et al., 2015; Farkona et al., 2016). 
The main reason for interest in an immunologic approach to treat cancer is that most traditional cancer treatments which include chemotherapy, radiotherapy, and surgery rely on drugs that kill dividing cells or block cell division, and these treatments have harmful effects on normal proliferating cells. As a result, the treatment of cancers causes significant morbidity and mortality. However, immune responses to tumors may be specific for tumor antigens and will not injure most normal cells. Therefore, immunotherapy has the potential of being the most tumor-specific treatment that can be devised (Farkona et al., 2016; Shore, 2015). The main aim of this paper is, therefore, to investigate the application of immunologic approaches to the treatment of cancer.

\section{Basics of Cancer Biology \\ Causes of Cancer}

Cancer is usually an acquired condition that typically occurs during the life of an individual (somatic mutations). At least $80 \%$ of all human cancers are related to exposure to agents that promote genetic changes in somatic cells. These environmental agents, such as UV light and certain chemicals, are mutagens that alter the DNA in a way that affects the function of normal genes (Ames \& Gold, 1998; Perera, 1996; Spitz \& Bondy, 2010; Zaridze, 2008). If the DNA is permanently modified in somatic cells, such changes may be transmitted during cell division. These DNA alterations can lead to effects on gene expression that ultimately affect cell division and thereby lead to cancer (Fig. 1) (Ponder, 2001). An environmental agent that causes cancer in this manner is called a carcinogen. Many types of cancer become more prevalent with age. The longer people live, the more exposure to carcinogens and the more time there is for genetic changes or mutations to occur within their cells. People who have weakened immune systems are more at risk of developing some types of cancer. This includes people who have had organ transplants and take drugs to suppress their immune systems to stop organ rejection, plus people who have HIV or AIDS, or other medical conditions which reduce their immunity to disease. Certain lifestyles and environmental factors are also known to cause mutations that can cause ancer (Ebomoyi, 2011; Zaridze, 2008).

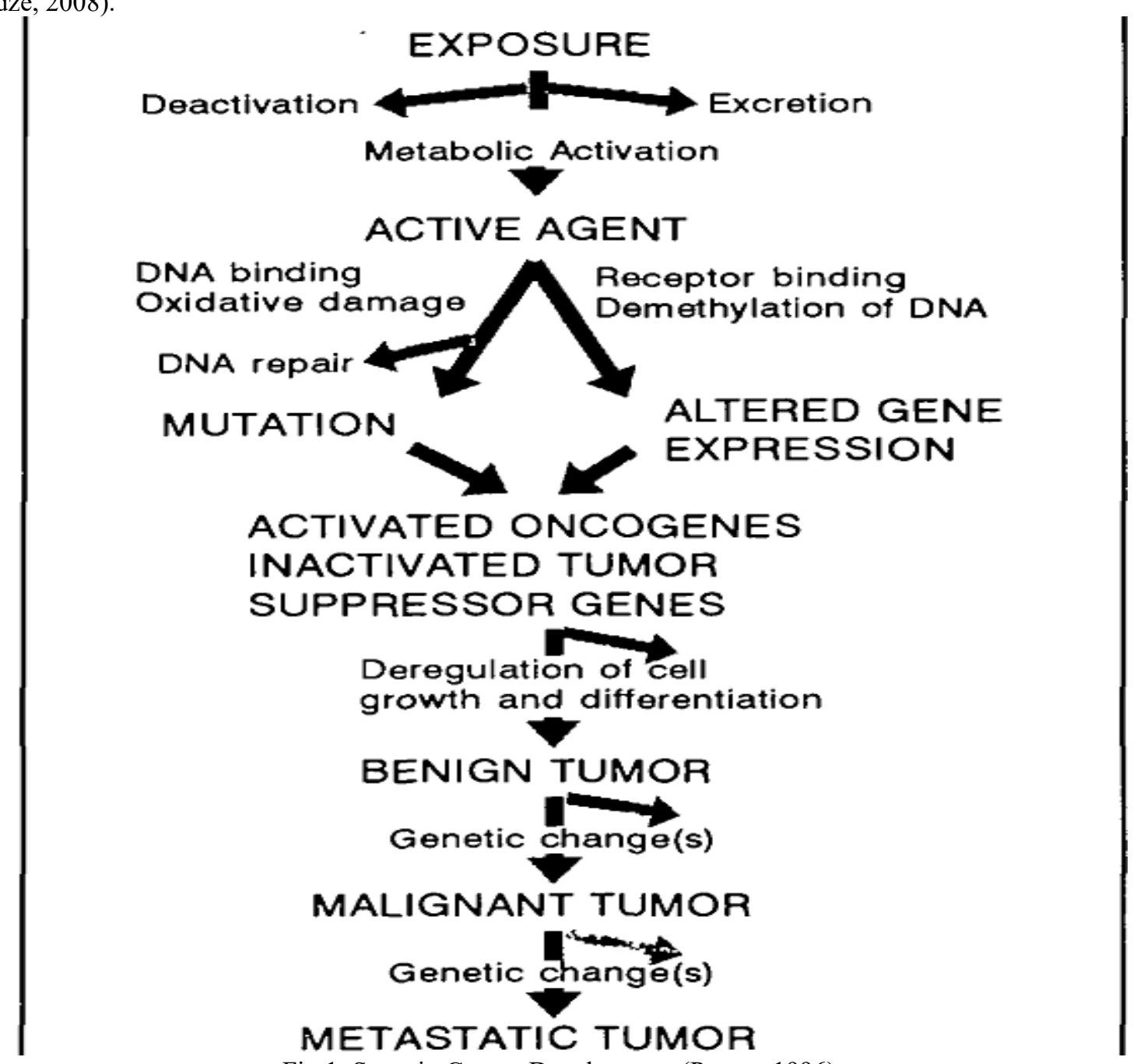

Fig 1. Steps in Cancer Development (Perera, 1996).

About $10 \%$ of human cancers are caused by viruses. Some researchers estimate that up to $15 \%$ of all human 
cancers are associated with viruses.

Retroviruses and viruses with double stranded DNA genomes cause tumors. Genes whose overactivity causes cells to become cancerous are called Oncogenes. Oncogenes commonly encode proteins that function in cell growth signaling pathways. These include growth factors, growth factor receptors, proteins involved in the intracellular signaling pathways, and transcription factors. They act in a genetically dominant fashion with respect to the unmutated (normal) version of the gene. Oncogenic retroviruses were the source of the first oncogenes to be isolated. Retroviruses become oncogenic either by expressing mutated versions of cellular growth-regulatory genes or by stimulating the overexpression of normal cellular genes with strong viral regulatory elements when integrate at an adjacent site (Ponder, 2001). Some Retroviruses carry oncogenes captured from host called proto-oncogenes, and transform the host cell by bringing the oncogene into the cell. For example, Rous sarcoma virus carries a mutated, oncogenic src gene that codes for an overactive tyrosine kinase. This enzyme is localized to the plasma membrane and phosphorylates the amino acid tyrosine in several cellular proteins which are essential in maintaining the cell's ability to respond to normal anti-growth signals from other cells and the extracellular matrix. When phosphorylated, they become active and override these signals, in effect signaling unregulated growth. The human retroviruses HTLV-1 and HTLV-2 transform a group of immune system cells called $\mathrm{T}$ cells by producing a regulatory protein that sometimes activates genes involved in cell division as well as stimulating virus reproduction.

The second transformation mechanism used by retroviruses involves the integration of a viral genome into the host chromosome such that strong, viral regulatory elements are near a cellular proto-oncogene. This results in such a high level of expression of the cellular protein. For example, some chicken retroviruses induce lymphomas when they are integrated next to the c-myc cellular proto-oncogene, which codes for a protein that is involved in the induction of either DNA or RNA synthesis (Brooker, 1998).

All known human dsDNA oncoviruses trigger cancerous transformation of cells by a similar mechanism. They encode proteins that bind to and thereby inactivate cellular proteins known as tumor suppressors. Tumorsuppressor proteins regulate cell cycling or monitor and/or repair DNA damage. Two tumor suppressors known to be targets of human oncovirus proteins are called Retinoblastoma (Rb) and p53 (Chen \& Hunter, 2005; Cooper and Hausman, 2007). Rb has multiple functions in the nucleus, all of which are critical to normal cell cycling. When $\mathrm{Rb}$ molecules are rendered inactive by an oncoviral protein, cells undergo uncontrolled reproduction. Thus they are said to be hyperproliferative. The protein p53 normally initiates either cell cycle arrest or programmed cell death in response to DNA damage. However, when p53 is inactivated by an oncoviral protein, it cannot do so and genetic damage persists. Cells can rapidly accumulate the additional mutations needed for oncogenic transformation (Karp, 2013).

Studies of viral oncogenes also led to the identification of cellular oncogenes or proto-oncogenes, which are involved in the development of non-virus-induced cancers. The key link between viral and cellular oncogenes was provided by studies of the highly oncogenic retroviruses. Most cancers occur, therefore, in the absence of viral infection. Genetic changes in proto-oncogenes convert them to oncogenes. A variety of oncogenes are activated by point mutations, DNA rearrangements, and gene amplification in human cancers (Karp, 2013).

About 5 to $10 \%$ of all cases of cancer involve inherited (germ-line) mutations (Ashton-prolla \& Vargas, 2014). These familial forms of cancer occur because people have inherited mutations from one or both parents that give them an increased susceptibility to developing cancer. This does not mean they will definitely get cancer but they are more likely to develop the disease than are individuals in the general population (Ames and Gold, 1998; Chen \& Hunter, 2005). Most inherited forms of cancer involve a defect in a tumor-suppressor gene (Petrucelli, Daly, and Feldman, 2010). In these cases, the individual is heterozygous, with one normal and one inactive allele. At the phenotypic level, a predisposition for developing cancer is inherited in a dominant fashion because a heterozygote exhibits this predisposition. The development of breast cancer for example shows an autosomal dominant pattern of inheritance with high penetrance in women (Ashton-prolla \& Vargas, 2014; Ebomoyi, 2011). Individuals with the disorder have inherited a loss-of-function mutation in the Breast cancer anigen 1 (BRCA-1) gene (Petrucelli et al., 2010). Most affected individuals have an affected parent. However, the actual development of cancer is recessive, because it initially relies on the loss of function of the normal copy of the BRCA-1 gene from a somatic cell (Kamps et al., 2017). This phenomenon is called loss of heterozygosity $(\mathrm{LOH})$, the loss of function of a normal allele when the other allele was already inactivated. It occurs in different ways. For example, the normal $B R C A-1$ gene could suffer a point mutation or a deletion that inactivates its function. A second way that tumor- suppressor genes are inhibited is via DNA methylation, particularly when it occurs in the vicinity of the promoter (Gruver et al., 2011; Mossman \& Scott, 2006; Rodríguez-paredes \& Esteller, 2011; Vineis \& Perera, 2007). Alternatively, the chromosome carrying the normal BRCA-1 gene could be lost during cell division. Another mechanism is mitotic recombination. When this occurs in a heterozygote, a daughter cell may receive two copies of the chromosome carrying the normal allele or two copies of the chromosome carrying the mutant allele. The cell carrying two copies of the mutant allele may continue to divide and accumulate additional genetic changes that result in breast cancer (Cooper and Hausman, 2007; Harris, 


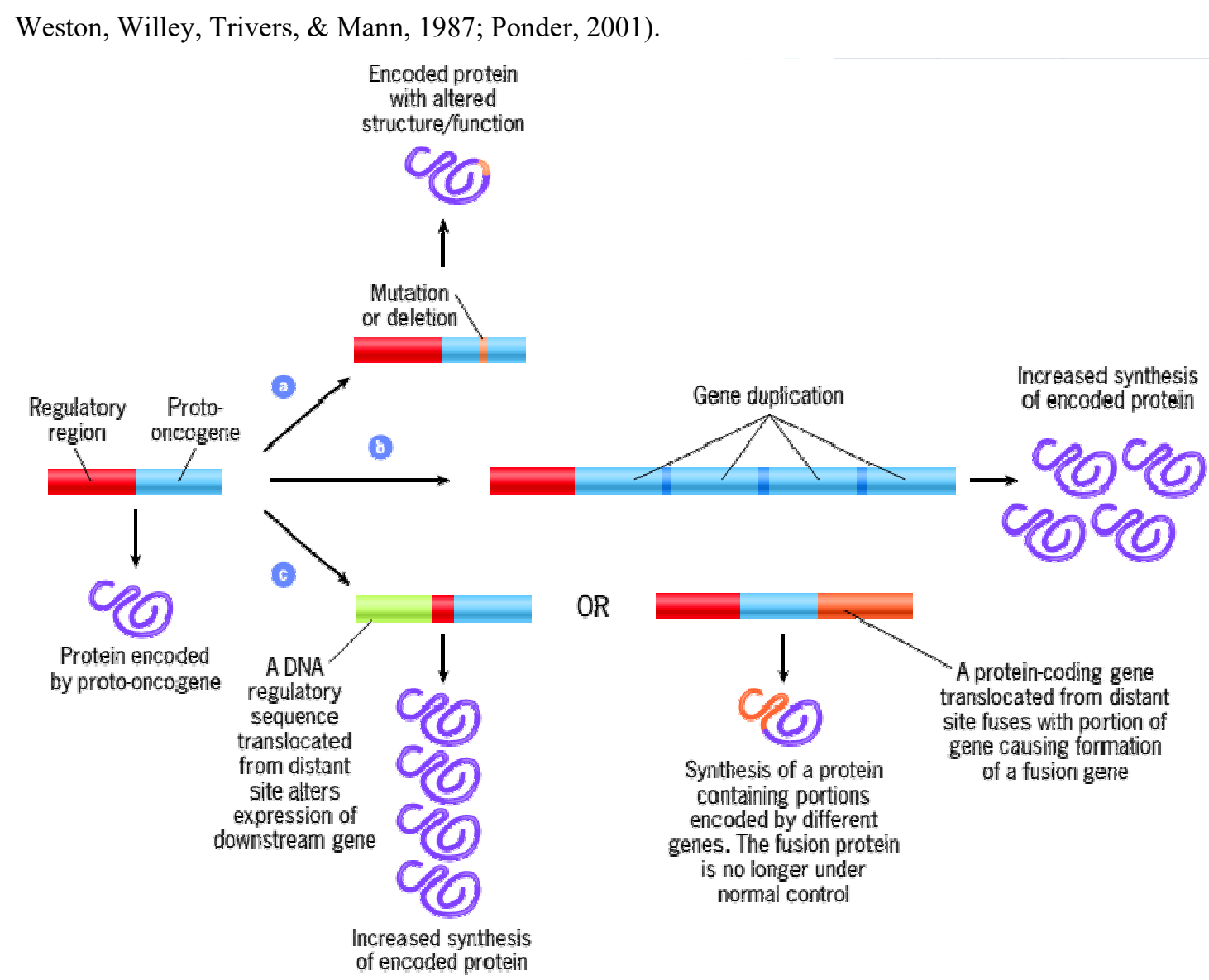

Fig 2.Activation of proto-oncogene to oncogene (Karp, 2013)

\section{Cancer and the Immune System}

Individual human tumors arise through a combination of genetic and epigenetic changes that facilitate immortality, but at the same time create foreign antigens, the so-called neo-antigens. Transduction of neoantigen expressing RNAs into antigen presenting cells, have led to success in triggering tumor-specific immune responses (Lu \& Robbins, 2016). The immune system is constantly scanning for mutations and malfunctions in cells that could lead to cancer, a process known as immunosurveillance (Lacombe, 2016; Li \& Song, 2017). It is established that natural killer (NK) cells, cytokines, dendritic cells (DCs), and T cells play important roles in the antitumor response (Fig.3). NK cells are a first line of defence against cancer. Cytokines contribute to the antitumor immune response in a variety of ways; they stimulate inflammation and boost the activation of other immune cells. DCs process and present antigens associated with tumors to T cells; this activates $\mathrm{T}$ cells to tumor associated antigen and initiates a targeted antitumor response. Importantly, recent studies demonstrate that $\mathrm{T}$ cell-based immunotherapies are also effective in a range of other human malignancies. However, because cancer is an immuno-evasive disease, adaptations that occur in the tumor microenvironment can blunt the immune response and contribute to cancer progression (Shore, 2015). Thus, tumors develop multiple resistance mechanisms, including local immune evasion, induction of tolerance, systemic disruption of $\mathrm{T}$ cell signaling and immune editing, which contributed for challenges in the development of cancer immunotherapies (Fig.3) (Abbas et al., 2015; Farkona et al., 2016; Finotello \& Trajanoski, 2017). As a result immunotherapies that boost the ability of endogenous T cells are required to destroy cancer cells (Schumacher \& Schreiber, 2015). 


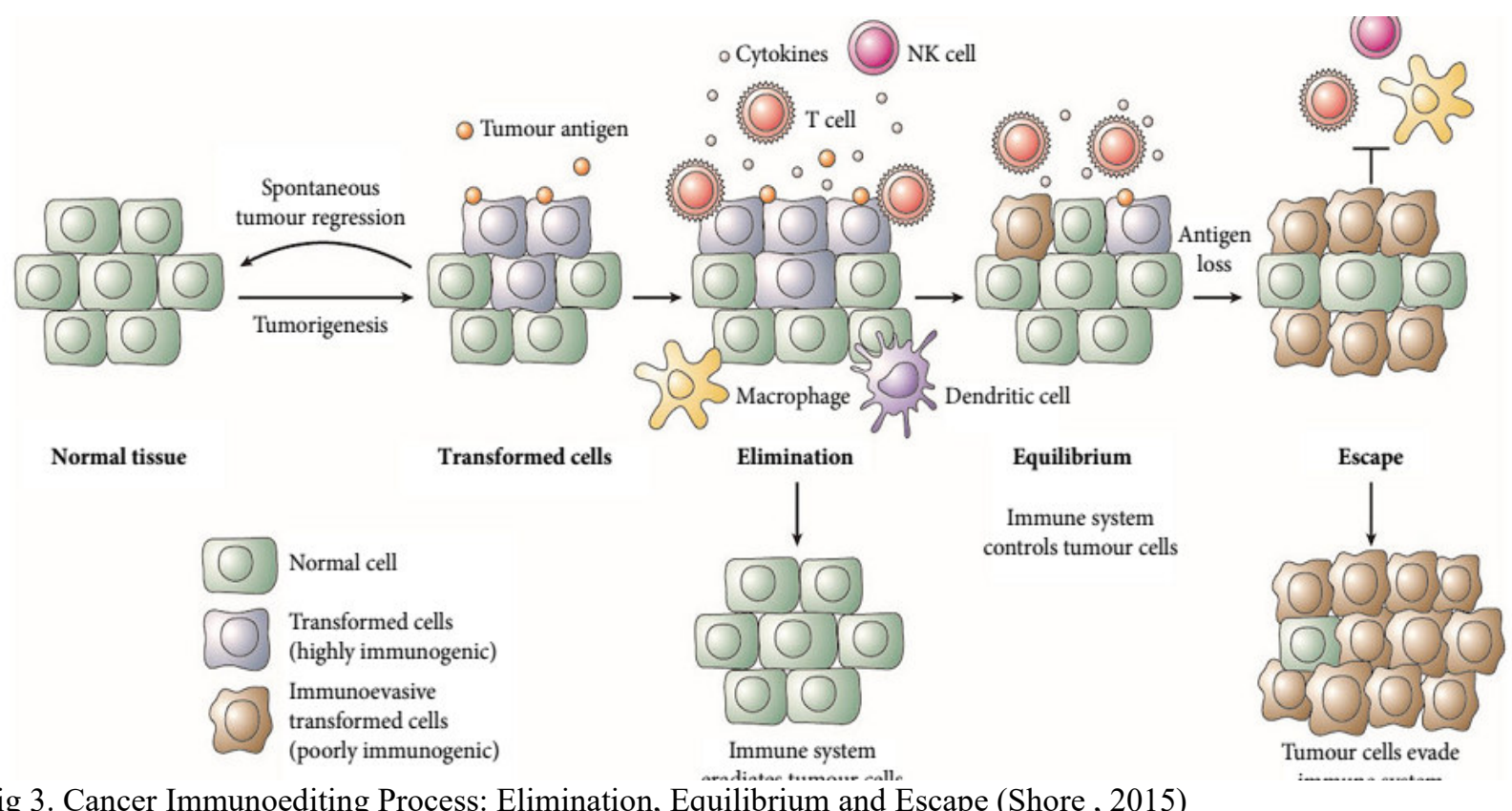

Fig 3. Cancer Immunoediting Process: Elimination, Equilibrium and Escape (Shore , 2015)

\section{Exploiting Cell Mediated Immune Responses to Cancer Immunotherapy}

The potential for treatment of cancer patients by immunologic approaches has held great promise for oncologists and immunologists for many years as most conventional therapies for cancer have harmful effect on normal proliferating cells. Therefore, immunotherapy has the potential of being the most tumor-specific treatment that can be devised (Yuzhakova et al., 2016).

Immunotherapy is a type of cancer treatment that is designed to help a person's immune system recognize and attack cancer cells. Immunotherapy is targeted at overcoming the suppression of immune surveillance, to allow recognition of tumor cells by the immune system and, as a result, the inhibition of tumor growth. Unlike classical methods of treatment of cancer immunotherapy is targeted at restoration of the ability of the immune system to fight the disease and not just at tumor removal with few side effects (Yuzhakova et al., 2016).

The earliest attempts to boost anti-tumor immunity relied on non-specific immune stimulation by cytokines, some bacterial products, synthetic molecules and hormones (Yuzhakova et al., 2016). Immune responses to tumors may be stimulated by the local administration of inflammatory substances or by systemic treatment with agents that function as polyclonal activators of lymphocytes. Non-specific immune stimulation of patients with tumors by injection of inflammatory substances such as killed bacillus Calmette-Guérin (BCG) at the sites of tumor growth has been tried for many years. The BCG mycobacteria activate macrophages and thereby promote macrophage-mediated killing of the tumor cells. In addition, the bacteria function as adjuvants and may stimulate $\mathrm{T}$ cell responses to tumor antigens. Intra-vesicular BCG is currently used to treat bladder cancer. Cytokine therapies, also, represent another method of enhancing immune responses in a non-specific manner (Sanghi et. al., 2014; Yuzhakova et al., 2016). Cytokines such as TNF- $\alpha$ (tumor necrosis factor), GMCSF (granulocytemacrophage colony stimulating factor), IFN- $\alpha$ (interferon alpha), as well as some interleukins including IL-2, IL7, IL-12, IL-15, IL-21 can act as agents for active immunotherapy. The effect of cytokines on the cells of the immune system include the stimulation of proliferation, differentiation, the activation and tracking of effector $\mathrm{CD} 4+$ and $\mathrm{CD} 8+\mathrm{T}$ lymphocytes and natural killers; the stimulation of proliferation and differentiation of progenitor cells of the hematopoietic system, which causes the formation of granulocytes, monocytes/macrophages and T lymphocytes; and the stimulation of synthesis by the immune system cells of IFN- $\gamma$ and immunoglobulins. Many cytokines also have the potential to induce non-specific inflammatory responses, which by themselves may have anti-tumor activity. The use of cytokines in anti-tumor immunotherapy demonstrated tumor regression, the prevention of metastasis formation, the development of immunological memory as well as a reduced risk of disease recurrence together with increased survival. The first cytokine, used for treating patients with leukemia and melanoma, was IFN- $\alpha$ and the second, for treating melanoma, was IL-2 (Yuzhakova et al., 2016).

More recently, tumor associated antigens are being developed to enhance immune response against the tumor (Lu \& Robbins, 2016; Yuzhakova et al., 2016). The identification of peptides recognized by tumorspecific CTLs and the cloning of genes that encode tumor-specific antigens recognized by CTLs have provided many candidate antigens to be included in tumor vaccines. For antigens that are unique to individual tumors, such as antigens produced by random point mutations in cellular genes, personalized vaccination approaches are 
now being attempted. An approach that has shown some success is the use of immunogenic long peptides that contain single amino acid changes corresponding to tumor mutations, along with selected adjuvants.

This approach, therefore, offers possibility for long-term cancer remission and decreases risk of recurrence by stimulating a long lasting immune guard against the cancer. It is highly selective for the cancer cells and can potentially be tailored to the patient's individual cancer, by the use of tumor-specific mutant antigens which are natural potential candidates for developing personalized cancer-specific vaccines (Circelli et al., 2015; Lacombe, 2016). Manipulation of the immune response has resulted in promising therapies by boosting the ability of the immune system to target cancer, or by limiting the ability of tumors to evade the natural immune response (Finotello \& Trajanoski, 2017). To be effective , immunotherapies need to increase the quality or quantity of immune effector cells, expose additional protective tumor neoantigens (i.e. antigen spread), and/or inhibit cancer -induced immunosuppressive mechanisms such as anti-cytotoxic T lymphocyte associated protein 4 (CTLA-4) antibody, ipilimumab, and of anti-programmed cell death protein 1 (PD1) PD-1 antibodies, $\mathrm{T}$ cell (or transmembrane) immunoglobulin and mucin domain protein 3 (Tim-3), depletion of Regulatory $\mathrm{T}$ cells and the use of antibodies targeting members of the tumor necrosis factor receptor superfamily so as to supply costimulatory signals to enhance T cell activity (Farkona et al., 2016; Li \& Song, 2017; Mahmood A, Srivastava A, Srivastava S, Pandya H, 2017; Phong et al., 2015; Schumacher \& Schreiber, 2015; Shore, 2015).

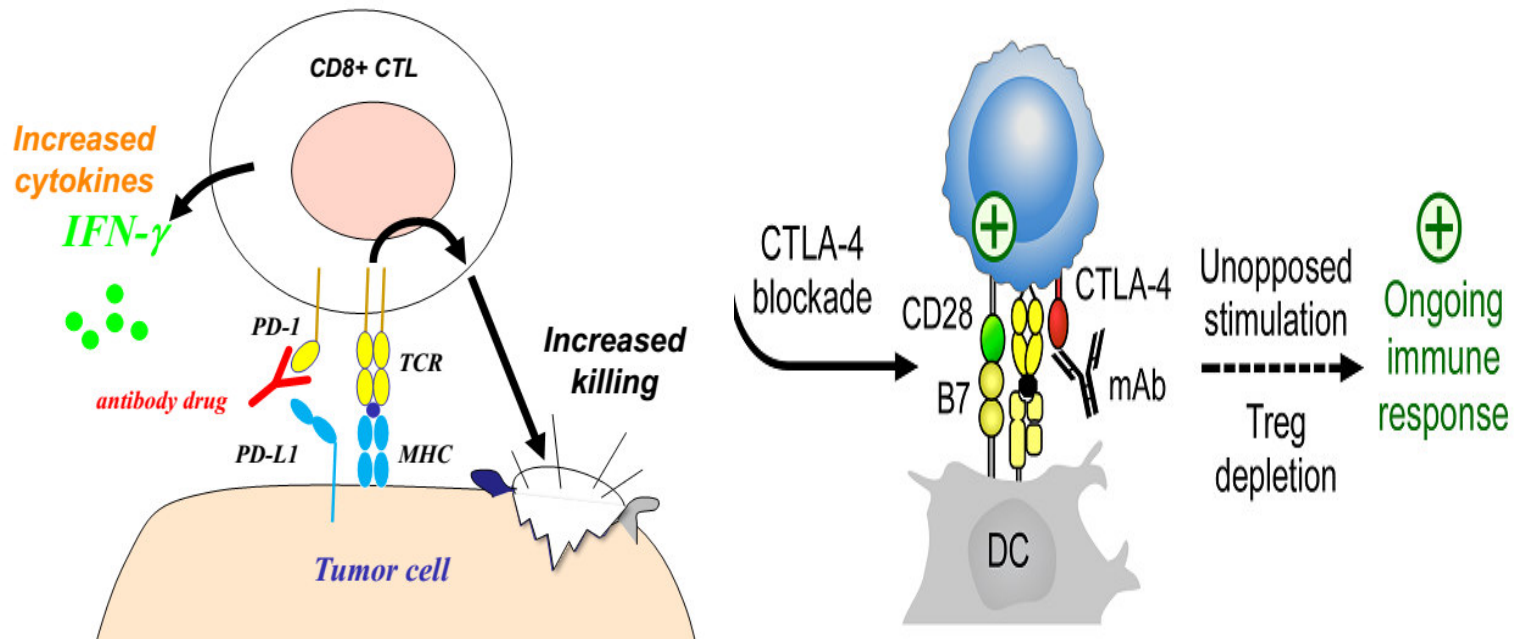

Fig.4. PD-1 or PD-L1 and CTLA-4 Blockade by mAbs Stimulates anti-tumor CTL response (Freeman, 2000)

Advances in our understanding of the immune system and in defining antigens on tumor cells have encouraged many new strategies. The different types of cancer immunotherapy include checkpoint inhibitors, vaccine immunotherapy, adoptive cell transfer and monoclonal antibodies and cytokines.

Cellular immunity mediated by $\mathrm{T}$ cell is strictly supervised and controlled by a check/balance system performed through many stimulatory and inhibitory molecules. The inhibitory receptors, also called immune checkpoints, regulate CTLs activation and effector function to maintain self-tolerance and limit bystander tissue damage as an indirect consequence of immune response against pathogenic invasion (Finotello \& Trajanoski, 2017; Yongshu et al., 2016). Currently, the most promising cancer immunotherapy approach is the blockade of immune checkpoints by using monoclonal antibodies that target PD-1 or PD-L1 and CTLA-4. This approach is based on the idea that tumor cells exploit various normal pathways of immune regulation or tolerance to evade the host immune response. Because these inhibitors establish checkpoints in immune responses, the approach of stimulating immune responses by removing inhibition is often called checkpoint blockade. An antibody specific for CTLA-4 (cytotoxic T-lymphocyte antigen-4), the inhibitory receptor on T cells for B7, is an approved therapy for advanced melanoma, and it is effective in slowing tumor progression in many patients. This antibody may work not only by blocking the action of CTLA-4 but perhaps also by depleting regulatory $\mathrm{T}$ cells, which express high levels of CTLA-4 (Farkona et al., 2016). A Study carried out by (Vétizou et al., 2015) indicated that the antitumor effects of CTLA-4 blockade depend on distinct Bacteroides species in the gut. T cell responses against tumors may also be inhibited by the PD-L1/PD-1 pathway. Antibody blockade of PD-1 or its ligand is effective in enhancing $\mathrm{T}$ cell killing of tumors in mice, and several human clinical trials have shown that PD-1 or PD-L1 blockade can limit tumor progression and reduce tumor burden in patients with advanced cancers. As they inhibit different phases of activation trials of combined blockade of both PD-1 and CTLA-4 appear to be very effective. TIM3 co-expression with PD1 on CD8+ tumor infiltrated T cells also indicated the need for combination therapies targeting both these pathways (Farkona et al., 2016). Common complications of these treatments have been autoimmune and inflammatory reactions. These reactions can be controlled with antiinflammatory medications such as corticosteroids. Furthermore, agents that block immune suppression and thereby boost anti-tumor immune responses may be most effective in combination with tumor vaccines (Couzin- 
Frankel, 2016; Farkona et al., 2016; Finotello \& Trajanoski, 2017; Lacombe, 2016; Lu \& Robbins, 2016; Yongshu et al., 2016).

Specific cancer associated antigens or viral antigens can be developed to treat cancers, and these are called vaccines. Cancer vaccines might be either therapeutic (used to treat existing cancers) or prophylactic (to prevent the development of cancer). Prophylactic vaccines that prevent infection with the virus are given to prevent infection-associated cancer (Farkona et al., 2016; Shore, 2015). The development of virally induced tumors, therefore, can be reduced by preventive vaccination with viral antigens or attenuated live viruses. The newly developed Hepatitis virus (HPV) vaccines (Gardasil for HPV associated cervical carcinoma) have been effective in decreasing the incidence of HPV-induced premalignant lesions in the cervix (Farkona et al., 2016; Yuzhakova et al., 2016).

Most tumor vaccines are therapeutic vaccines; obtained from tumor associated antigens and they have to be given after the host has encountered the tumor and in order to be effective, they have to overcome the immune regulation that cancers establish (Farkona et al., 2016; Yuzhakova et al., 2016). Therapeutic vaccines based on tumor-associated antigens contain either these particular antigens in the form of peptides of the proteins, or dendritic cells (DCs) loaded with tumor-associated antigens, or autologous or allogenic tumor cells carrying the complete set of tumor associated antigens or a mixture of DCs and tumor cells (Yuzhakova et al., 2016). DCs are known to be the most effective antigen presenting cells and play a pivotal role in coordinating innate and adaptive immune responses. Thus, for cancer vaccines to break the tolerance, high quantities of antigens must be targeted to DCs and these, in turn, need to be expanded and activated with appropriate agents (Farkona et al., 2016).

The mechanism of action for therapeutic cancer vaccines involves activating the immune system with targeted $T$ cells to seek out and destroy target cancer cells (Farkona et al., 2016). The therapeutic vaccine Sipuleucel-T (Provenge) an autologous cellular immunotherapy, based on DCs has been approved by the Food and Drug Administration (FDA, USA) in 2010 as the first cellular immunotherapy for prostate cancer treatment. This vaccine was designed to induce an immune response targeted against prostatic acid phosphatase (PAP), an antigen expressed in most prostate cancers. During ex vivo culture with PAP-granulocyte- macrophage colonystimulating factor (GM-CSF), DCs take up and process the recombinant target antigen into small peptides that are then displayed on the APC surface (Farkona et al., 2016). Proinflammatory molecules are used to enhance the numbers of activated dendritic cells at the vaccination site. These adjuvants include toll like receptor (TLR) ligands, such as dsRNA, CpG DNA, and BCG, and cytokines such as GM-CSF and IL-12. In general, cellmediated immunity to tumors can theoretically be enhanced by treating tumor-bearing individuals with cytokines that stimulate the proliferation and differentiation of T lymphocytes and NK cells (Fig.3) (Shore, 2015). GMCSF promotes the maturation of dendritic cells, which present the tumor antigen and stimulate anti-tumor T cell responses. Once infused back into patients, these activated DCs can interact with both CD4 T cells and CD8 T cells and initiate downstream immune responses (Fig.6)(Cubey, 2002).

Another approach is the use of DNA vaccines and viral vectors encoding tumor antigens; some of these are in active or planned clinical trials. The cell-based and DNA vaccines may be the best ways to induce CTL responses because the encoded antigens are synthesized in the cytosol of cells, such as dendritic cells, that take up these antigens, plasmids and vectors, and peptides enter the class I MHC pathway of antigen presentation (Karp, 2013).

There are several important characteristics of therapeutic cancer vaccines, which may make this newer type of immunotherapy an attractive treatment option. First, therapeutic vaccines directly target the immune system, which subsequently expands the immune system's attack on tumor cells. Second, because the immune response is generated over time, the observed effect of therapeutic vaccines is not immediate. Furthermore, by engaging the immune system, therapeutic vaccines may create immunological memory that can extend protection beyond the immediate treatment period. Finally, because the immune response may evolve over time, a broadening of the immune response may be observed such that while the immune system continues to attack the original tumor target, it might also evolve to attack additional tumor- specific antigens (antigen spread) (Shore, 2015; Yuzhakova et al., 2016). However, the results of trials with many different types of tumor vaccines have been inconsistent, which reflects one of the major characteristics of to evade host immunity. The combination of vaccination with other immunotherapies such as immune inhibitor receptors blockade had showed a great improvement (Farkona et al., 2016). 


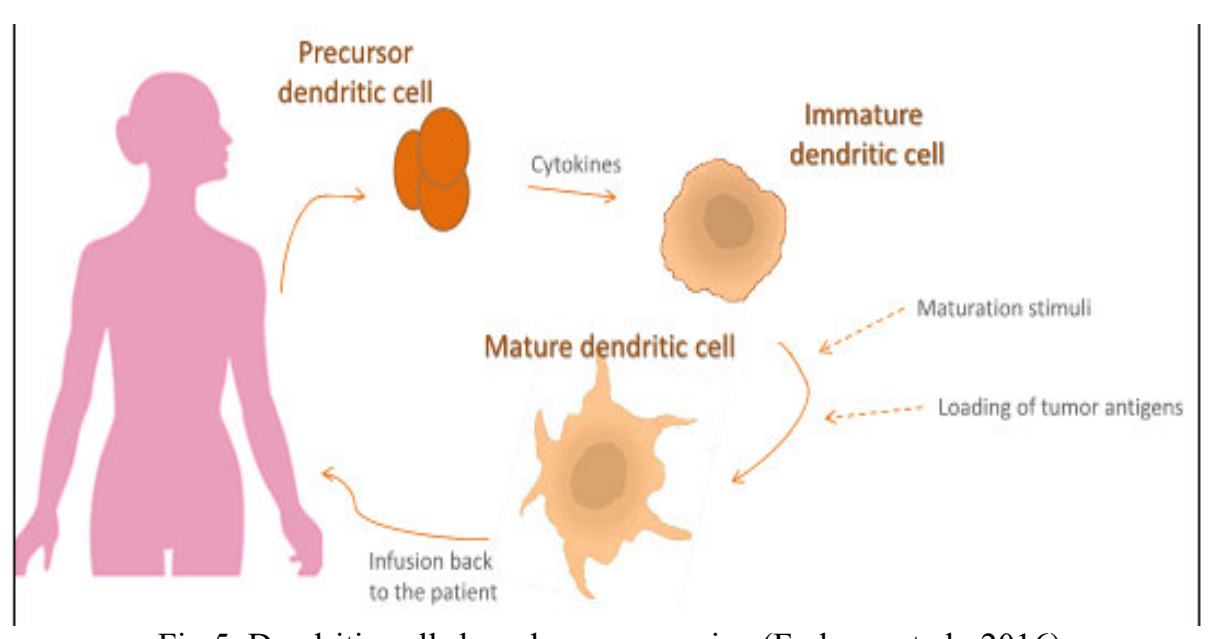

Fig 5. Dendritic cells based cancer vaccine (Farkona et al., 2016)

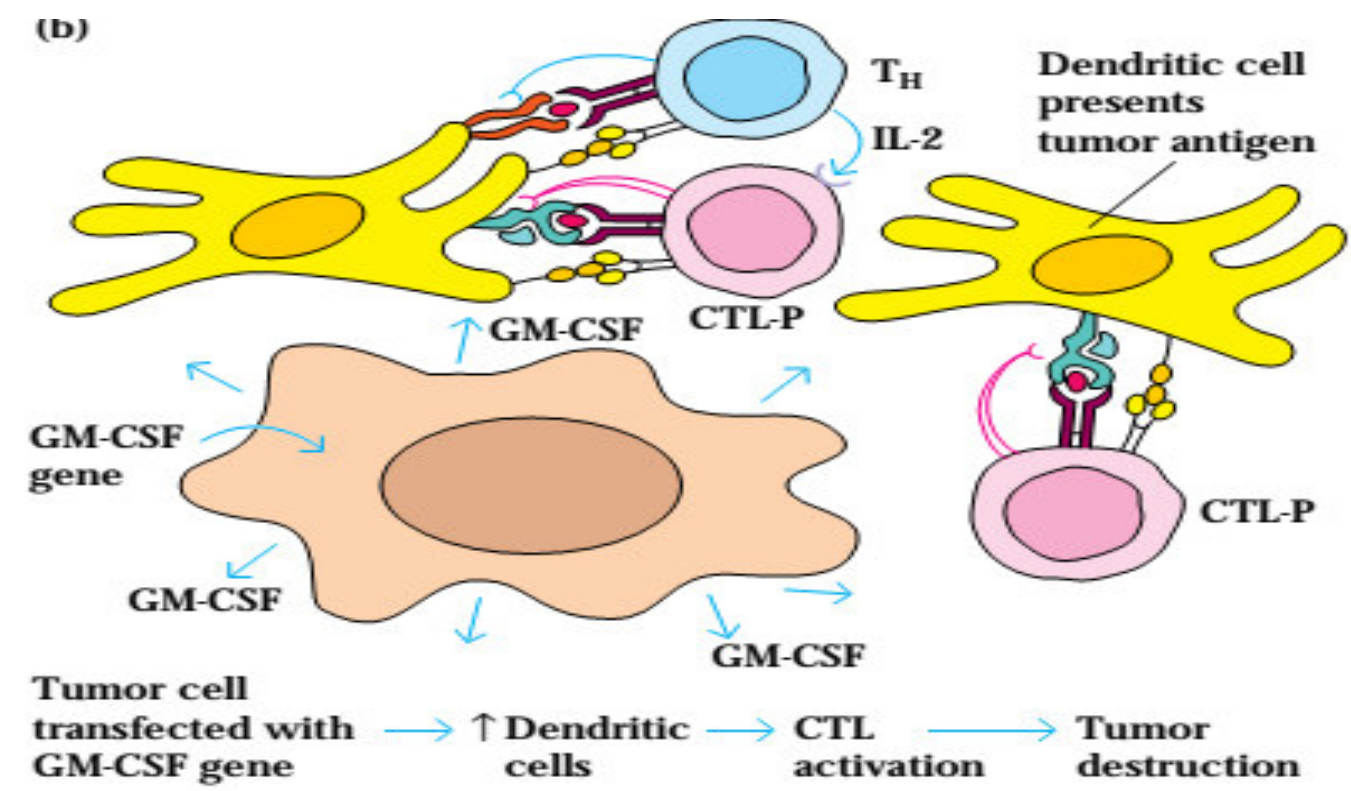

Fig. 6. Activation of dendritic cells to present tumor antigens to both TH cells and CTL-(Cubey,2002).

Some viruses are being used to boost immune activity in cancer patients. These oncolytic (cancer bursting) viruses act in two ways; they kill some cancer cells directly and activate the immune system. After the viral lysis of tumor cells, tumor associated antigens are released within the vicinity of the tumor, resulting in enhanced CD8+ $\mathrm{T}$ cell-mediated antitumor responses. A herpes virus called Talimogene laherparepvec (T-VEC) has recently been approved for use in melanoma and many other viruses are being tried in laboratory and clinical studies (Farkona et al., 2016; Lacombe, 2016).

Some cancers, such as breast cancer and lymphomas, display certain molecules on their surface at very high levels which targeted by monoclonal antibodies. The use of antibodies for treatment of cancer is limited to extracellular targets, such as growth factors or cell surface receptors. A humanized monoclonal antibody, Herceptin is currently being used to treat a form of breast cancer (Lacombe, 2016). Herceptin specifically neutralizes a genetically determined growth factor, HER2 that promotes the proliferation of the cancer cells. It is expressed in relatively high quantities in about $25-30 \%$ of breast cancer patients. Erbitux, a monoclonal antibody against the epiderma groth factor (EGF) receptor (the ErbB oncogene protein), was also approved by the FDA in 2004 for use in treatment of advanced colorectal cancer (Yuzhakova et al., 2016). These antibodies carryout their effective function through capsonization, complement activation or antibody dependent cell cytotoxicity.

The transfer of cultured immune cells that have anti-tumor reactivity into a tumor-bearing host known as adoptive $\mathrm{T}$ cell therapy (ACT) is a promising form of immunotherapy which exploits the antitumor properties of lymphocytes to eradicate primary and metastatic tumor cells (Farkona et al., 2016). Agents for ACT include: lymphokine-activated CD8+ T lymphocytes (lymphokine-activated killer, LAK), obtained from peripheral mononuclear blood cells after cultivation in vitro in the presence of IL-2; CD8+ T lymphocytes obtained from 
peripheral mononuclear blood cells in vitro with the assistance of several factors including IL-2, IL-1, IFN- $\gamma$ and anti-CD3 monoclonal antibodies (cytokine-induced killer, CIK); CIK, cultivated in the presence of DC (DC$\mathrm{CIK}$ ); and tumor-infiltrating lymphocytes (tumor-infiltrating lymphocyte, TIL), obtained from patients with tumors (Yuzhakova et al., 2016).

Lymphocytes are derived from cancer patients' peripheral blood or solid tumor tissue, and then are treated in various ways to expand their numbers and enhance their anti-tumor activity before re-infused back into the patient. ACT with tumor-infiltrating lymphocytes (TILs) is an approach where T cells, generally mixtures of CD8+ and CD4+ T cells grown from resected metastatic tumor deposits, are harvested and expanded ex vivo prior to adoptive transfer. This approach attempts to reverse the functional impairment of the tumor-specific $\mathrm{T}$ cells that reside within the tumor, and caused by the immune suppressive tumor microenvironment, by growing them prior to the reinfusion in a cocktail of various cytokines and tumor-specific mutant antigens (Circelli et al., 2015). The ability of adoptive immunotherapy to mediate durable tumor regressions in some patients may depend in part upon the fact that many TIL populations contain T cells that recognize multiple neoantigens (Lu \& Robbins, 2016; Schumacher \& Schreiber, 2015; Yuzhakova et al., 2016) or driver mutations that are essential for carcinogenesis. Host lymphodepletion before TIL infusion is speculated to improve TIL functionality by eliminating immunosuppressive cells, such as Treg and myeloid-derived suppressor cells (MDSCs), in the tumor microenvironment (Farkona et al., 2016).

$\mathrm{T}$ cells can be genetically modified so that they more readily recognize the tumor. Chimeric antigen receptor (CAR) T cells consist of an Immunoglobulin (Ig) variable domain fused to a TCR constant domain. CARs are genetically engineered receptors with tumor antigen-specific binding sites encoded by Ig-variable genes and cytoplasmic tails containing signaling domains of both antigen receptors and costimulatory molecules. CAR therapy is a personalized treatment that involves genetically modifying a patient's $\mathrm{T}$ cells to make them target tumor cells (Benjamin, Cox, Platt, \& Zhang, 2015). The advent of CARs omit s the need for tumor cells to carry a functional antigen processing machinery or to express antigens through MHC class molecules since the engineered $\mathrm{T}$ cells obtain the antigen recognition properties of antibodies.

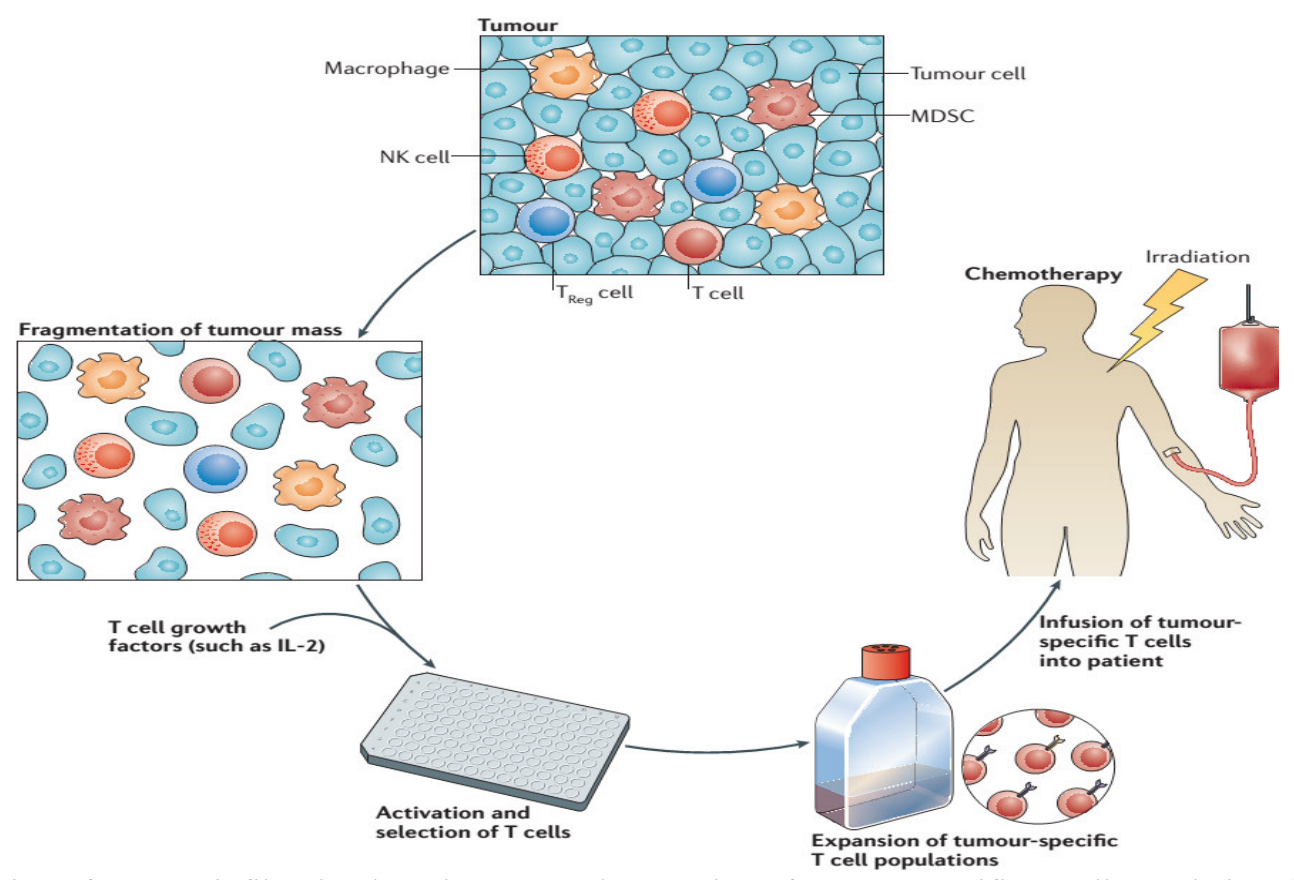

Fig.7. Isolation of tumour-infiltrating lymphocytes and expansion of tumour-specific T cell populations(Nicholas P. Restifo, 2012)

CAR therapy is a personalized treatment that involves genetically modifying a patient's $\mathrm{T}$ cells to make them target tumor cells (Benjamin, Cox, Platt, \& Zhang, 2015). The advent of CARs omit s the need for tumor cells to carry a functional antigen processing machinery or to express antigens through MHC class molecules since the engineered $\mathrm{T}$ cells obtain the antigen recognition properties of antibodies. Tumor regression following administration of genetically engineered $\mathrm{T}$ cells has been observed in B-cell malignancies, melanoma, and synovial sarcoma (Couzin-Frankel, 2013; Farkona et al., 2016; Lacombe, 2016; Schumacher \& Schreiber, 2015). 


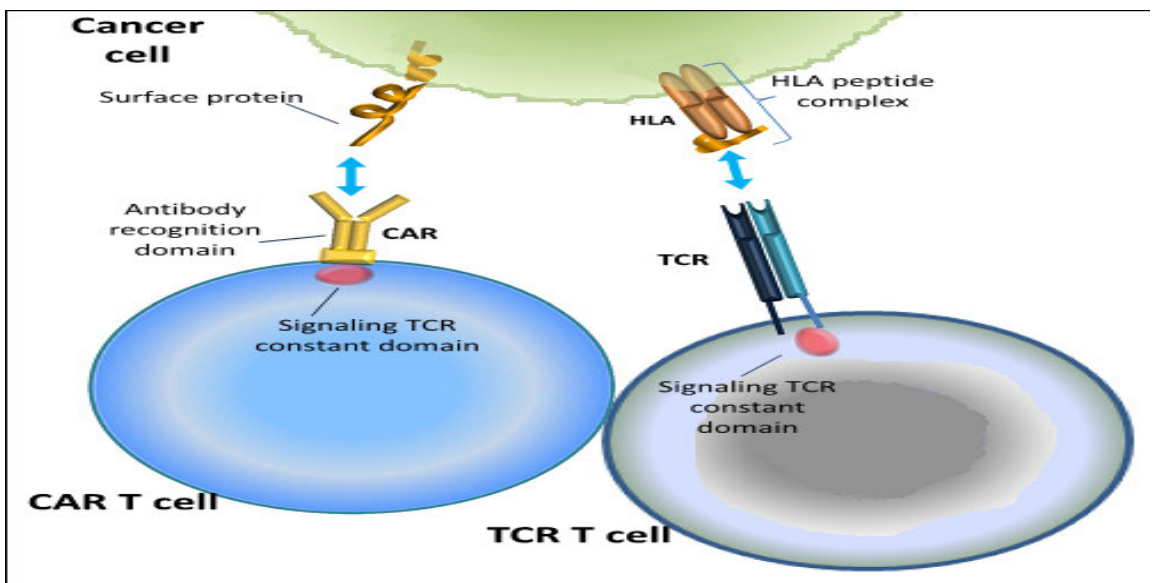

Fig.8. Genetic $\mathrm{T}$ cell engineering for the improvement and broadening of tumor-infiltrating lymphocyte (TIL) therapy (Farkona 2016)

The Roles of Stem Cell Memory T Cells in Cancer Immunotherapy

Upon antigen recognition, naive $\mathrm{T}$ cells $(\mathrm{TN})$ undergo extensive functional and phenotypic changes that drive their differentiation into effectors and memory cells. The memory compartment is multi-faceted and encompasses multiple $\mathrm{T}$ cell subsets with divergent properties(Cieri et al., 2017). Memory T cells include several subtypes: central memory (TCM), effector memory (TEM), and terminal effector (TTE) T cells. More recently, stem cell memory T (TSCM) cells have been described as a new immune biomarker for evaluating long-term memory $\mathrm{T}$ cell immune reconstitution, which is an important index after hematopoietic stem cell transplantation (HSCT) (Cieri et al., 2017; Hosokawa et al., 2017; Xu, Zhang, Luo, \& Li, 2015). TSCM cells have been shown to be able to differentiate into central memory T cells (TCM), effector memory (TEM), and terminal effector T cells (TTE). They have been described in mice, non-human primates and in humans, constituting approximately $2-4 \%$ of the total $\mathrm{CD} 4^{+}$and $\mathrm{CD} 8^{+} \mathrm{T}$-cell population in the periphery. TSCM represent the earliest and long-lasting developmental stage of memory $\mathrm{T}$ cells, displaying stem cell-like properties, and exhibiting a gene profile between navve and central memory $\mathrm{T}$ cells. Their self-renewal capacity and long-term survival has sparked interest to employ them in the cancer immunotherapy(Flynn \& Gorry, 2014). TSCM have shown properties of self-renewal and superior antitumor responses compared with other memory T-cell subsets in adoptive T-cell therapy studies in mice(Nicholas, Restifo, 2012,Xu et al. 2015). According to the study of Xu et al. (2015) these characteristics may be able to reduce the current therapeutic limitations of adoptive immunotherapies including poor T-cell engraftment, poor persistence and an inability to mount a long lasting immune response. Adoptively transferred $\mathrm{CD}^{+} \mathrm{T}$ memory stem cells (TSCM), the earliest differentiated circulating memory T-cell population possessing superior stem cell-like qualities identified thus far, preferentially survived in vivo compared with more differentiated central memory (TCM) or effector memory T (TEM) cells in patients treated with genetically modified lymphocytes (Cieri et al., 2017; Flynn \& Gorry, 2014; Nicholas P. Restifo, 2012; Pilipow et al., 2015). TSCM cells have a genetic programme that enables them to proliferate extensively, and they can further differentiate into TCM and TEM cells. Importantly, human T SCM cells show increased antitumour activity compared with TCM and TEM cells, suggesting that they will be more effective for ACT in patients with cancer(Fig.10)(Nicholas, Restifo, 2012).The TSCM seem therefore the ideal subset to exploit to induce long-lasting antitumor T-cell responses. In vivo, TSCM develop from naïve T cells (TN) cell precursors following transfer in lymphodepleted hosts harboring increased levels of plasma IL15 and IL7 (Gao, Zhao, Wan, \& Zhu, 2015; Pilipow et al., 2015). Studies demonstrated that the TSCM cells persisted and preserved their precursor potential in humans for up to 12 years after the infusion of gene corrected stem cells (Xu et al., 2015).Since TSCM cells were shown to be capable of reconstituting the full repertoire of memory and effector T cells after HSCT, it is particularly attractive to use them for the design and development of effective vaccines as well as T cell-based therapies. Even though potent antitumor activity of TSCM cells was demonstrated in preclinical animal tumor models, it is currently not feasible to treat patients with naturally occurring TSCM cells (Cieri et al., 2017) due to their low number in circulating lymphocytes. However, it was shown that a large number of TSCM cells were generated by priming naive T cells with low doses of IL-7 and IL-15(Cieri et al., 2017). It is therefore possible to generate, expand, and genetically engineer TSCM cells in vitro from naive precursors to use in cancer immunotherapy. 


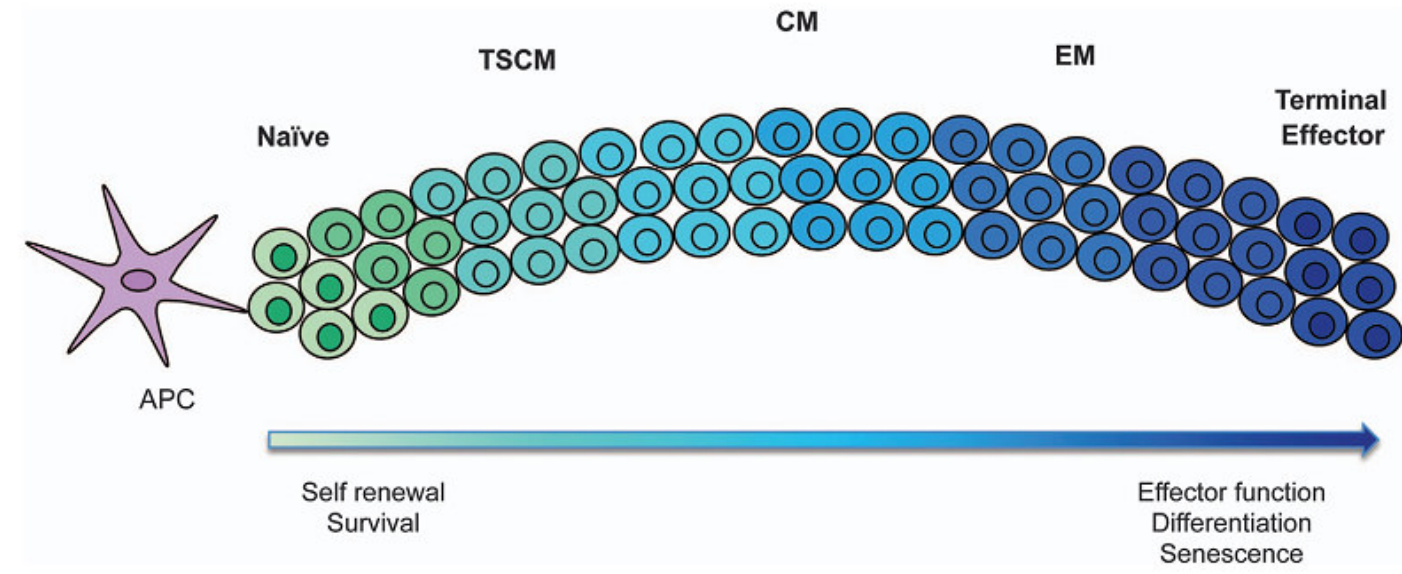

Fig.9. Following the theory of a linear hierarchical system, upon antigen exposure, nar"ve T cells proliferate and differentiate into memory T cells (Flynn \& Gorry, 2014)
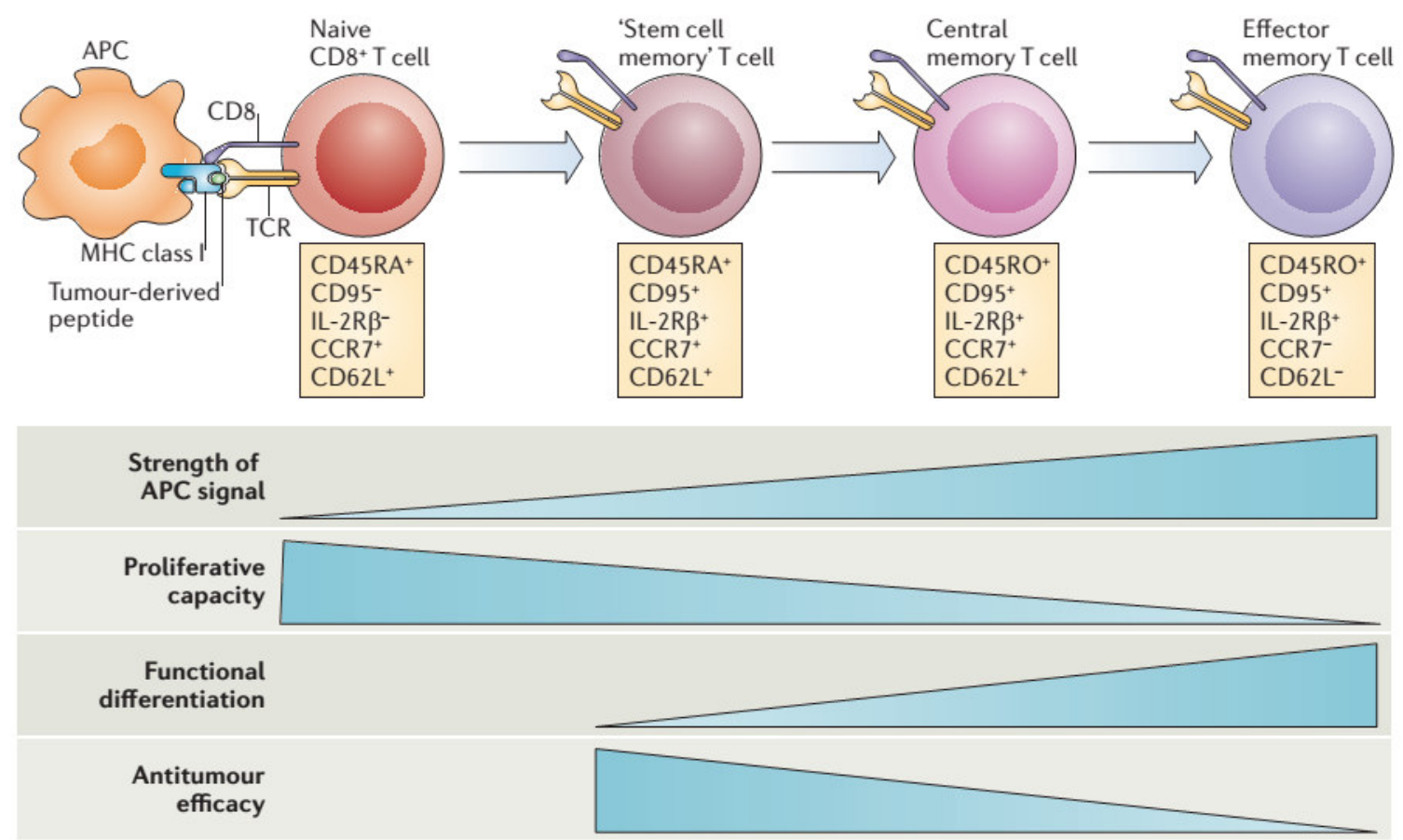

Fig. 10 Progressive $\mathrm{T}$ cell differentiation diminishes proliferative and antitumour capacities. The process of $\mathrm{T}$ cell differentiation results in the loss of proliferative and self-renewal capacity. (Nicholas Restifo, 2012)

\section{Conclusion}

Cancer is a disease characterized by uncontrolled cell division. It results from alterations in regulatory genes that control cell proliferation, differentiation, and survival.

Cancer is the major cause of mortality and morbidity worldwide. The current treatment strategies target both tumors and normal dividing body cells has harmful side effects and is not effective particularly at metastatic stage. The recently developed promising strategy is the use of cellular immunotherapy. At the beginning, the immune system can recognize tumor associated antigens as foreign and destroy transformed cells. However, cancer cells eventually develop mechanisms to escape control by the immune system, leading to disease progression. The activity of the immune system of the patient can be boosted or restored by stimulating the immune system non- specifically by using pro inflammatory agents such cytokines and bacterial products. The cellular immune response to tumor can also be enhanced through activation by cancer associated antigens, by in vitro activation and reinfusion of dendritic cells with tumor antigens, blockade of the immune blockers in vitro cultivation and transfer of $\mathrm{T}$ cells and memory stem $\mathrm{T}$ cells. Furthermore, a combination therapy that involves administration of two immunotherapies is found to be more effective than monotherapy based on the correct combinations. 


\section{References}

Aaron M. Gruver, Bryce P. Portier, ; Raymond R. Tubbs. (2011). Molecular Pathology of Breast Cancer. Arch Pathol Lab Med, 135, 544-557.

Abbas, A. K., LicHtman, A. H., \& Pillai, Sh. (2015). Immunity to Tumors. In Cellular and Molecular Immunology 8th edition (pp. 383-396).

Ames, B. N., \& Gold, L. S. (1998). The Causes and Prevention of Cancer : The Role of Environment. Biothera, $11,205-220$.

Ashton-prolla, P., \& Vargas, F. R. (2014). Prevalence and impact of founder mutations in hereditary breast cancer in Latin America. Genet and Mol Biol, 37(1), 234-240.

Benjamin, D., Cox, T., Platt, R. J., \& Zhang, F. (2015). Therapeutic genome editing : prospects and challenges. Nature , 21(2), 121-131.

Brooker, R. J. (1998). MEDICAL GENETICS AND CANCER. In Genetic Analysis and Principles (pp. 614630).

Chen, Y., \& Hunter, D. J. (2005). Molecular Epidemiology of Cancer. Cancer 55(1), 45-54.

Cieri, N., Oliveira, G., Greco, R., Forcato, M., Taccioli, C., Lunghi, F., ... Bordignon, C. (2017). Generation of human memory stem $\mathrm{T}$ cells upon haploidentical T-replete hematopoietic stem cell transplantation. Blood, $125,2865-74$

Circelli, L., Petrizzo, A., Tagliamonte, M., Tornesello, M. L., Buonaguro, F. M., \& Buonaguro, L. (2015). Systems Biology Approach for Cancer Vaccine Development and Evaluation. Vaccines, 3, 544-555.

Couzin-Frankel, J. (2013). Cancer Immunotherapy. Sci, 8342(6165), 1432-1433.

Cubey. (2002). Immunology.

Ebomoyi, E. W. (2011). Genomic Epidemiology of BRCA1 / BRCA2 ; Breast Cancer Associated Genes and Use of Electronic Health Record to Reduce the Escalating Cost of Treatment. Brit JMed \& Med Res, 1(4), 333345.

Farkona, S., Diamandis, E. P., \& Blasutig, I. M. (2016). Cancer immunotherapy: the beginning of the end of cancer? BioMed C Med, 14(73), 1-18. 0623-5

Finotello, F., \& Trajanoski, Z. (2017). New strategies for cancer immunotherapy: targeting regulatory T cells. Gen Med, 9, 10-12.

Flynn, J. K., \& Gorry, P. R. (2014). Stem memory T cells ( TSCM ) - their role in cancer and HIV immunotherapies. Clin \& Transl Immunol, 3(7), e20-7. Freeman, G. (2000). PD-1 Cancer Immunotherapy. J. Exp. Med., 192(7), 1-73.

Gao, J., Zhao, L., Wan, Y. Y., \& Zhu, B. (2015). Mechanism of Action of IL-7 and Its Potential Applications and Limitations in Cancer Immunotherapy. Int. J. Mol. Sci., 16, 10267-10280.

Geoffre M. Cooper and Robert E. Hausman. (2007). Cancer. In The Cell A Molecular Approach 4th edition (pp. 719-800).

Harris, C. C., Weston, A., Willey, J. C., Trivers, G. E., \& Mann, D. L. (1987). Biochemical and Molecular Epidemiology of Human Cancer: Indicators of Carcinogen Exposure, DNA Damage , and Genetic Predisposition. Environ Health Perspectives, 75(10), 109-119.

Hosokawa, K., Muranski, P., Feng, X., Townsley, M., Liu, B., Knickelbein, J., ... Neal, S. (2017). Memory Stem T Cells in Autoimmune Disease: High Frequency of Circulating CD8 + Memory Stem Cells in Acquired Aplastic Anemia. J Immunol, 196, 1-12.

Kamps, R., Brandão, R. D., Bosch, B. J. Van Den, Paulussen, A. D. C., Xanthoulea, S., Blok, M. J., \& Romano, A. (2017). Next-Generation Sequencing in Oncology: Genetic Diagnosis , Risk Prediction and Cancer Classification. Int J Mol Sci, 18(308). Karp, G. (2013). Cancer. In Cell and Molecular Biology Concepts and experiments (pp. 664-669).

Lacombe, S. (2016). Cancer Immunotherapy. British society for Immunology.

Li, D., \& Song, S. (2017). Engineer Immune Cells to Fight Cancer. J Stem Cell Res Ther, 2(3), 3-4.

Lu, Y., \& Robbins, P. F. (2016). Targeting neoantigens for cancer immunotherapy. Int Immunol, 28(7), 365-370. http://doi.org/10.1093/intimm/dxw026

Mahmood A, Srivastava A, Srivastava S, Pandya H, K. N. (2017). Role of Cell Based Approaches in Cancer Immunotherapy. J Stem Cell Res Ther, 2(5), 2-5.

Mossman, D., \& Scott, R. J. (2006). Epimutations , Inheritance and Causes of Aberrant DNA Methylation in Cancer. Hered Cancer in Clin Pract, 4(2), 75-80.

Nicholas P. Restifo, M. E. D. and S. A. R. (2012). Adoptive immunotherapy for cancer: Adoptive immunotherapy for cancer : harnessing the T cell response. Nature, 12(4), 269-281.

Perera, F. P. (1996). Molecular Epidemiology : Insights Into. J Nat Cancer Inst, 88(8).

Petrucelli, N., Daly, M. B., \& Feldman, G. L. (2010). Hereditary breast and ovarian cancer due to mutations in BRCA1 and BRCA2. Genet Med, 12(5), 245-259.

Phong, B. L., Avery, L., Sumpter, T. L., Gorman, J. V, Watkins, S. C., Colgan, J. D., \& Kane, L. P. (2015). Tim- 
3 enhances Fc $\varepsilon$ RI-proximal signaling to modulate mast cell activation. J. Exp. Med., 3(88), 1-16.

Pilipow, K., Roberto, A., Roederer, M., Waldmann, T. A., Mavilio, D., \& Lugli, E. (2015). IL15 and T-cell Stemness in T-cell - Based Cancer Immunotherapy. Cancer Res, 75((24), 5187-5194.

Ponder, B. A. J. (2001). Cancer genetics. Nature, 411(336-342).

Rodríguez-paredes, M., \& Esteller, M. (2011). Cancer epigenetics reaches mainstream oncology. Nature , 17(3), 330-339.

Sanghi D. K. et. al. (2014). A detail comprehensive review on vaccines. Int. J. Res. Dev. Pharm. L. Sci, 3(2), 887-895.

Schumacher, T. N., \& Schreiber, R. D. (2015). Neoantigens in cancer immunotherapy. Sci, 348(6230), 69-76.

Shore, N. D. (2015). Advances in the understanding of cancer immunotherapy. BJU Int, 321-329.

Spitz, M. R., \& Bondy, M. L. (2010). The evolving discipline of molecular epidemiology of cancer. Carcinogenesis, 31(1), 127-134.

Vétizou, M., Pitt, J. M., Daillère, R., Lepage, P., Waldschmitt, N., Flament, C., ... Schlitzer, A. (2015). Anticancer immunotherapy by CTLA-4 blockade relies on the gut microbiota. Sci, 350(6264), 1079-1084.

Vineis, P., \& Perera, F. (2007). Molecular Epidemiology and Biomarkers in Etiologic Cancer Research: The New in Light of the Old. Cancer Epidemiol Biomarkers Prev, 16(10), 1954-65.

Xu, L., Zhang, Y., Luo, G., \& Li, Y. (2015). The roles of stem cell memory T cells in hematological malignancies. J Hematol \& Oncol, 8(113), 10-14.

Yongshu Li ., Fangfei Li., Feng Jiang, Xiaoqing Lv., Rongjiang Zhang, Aiping Lu ., and G. Z. (2016). A MiniReview for Cancer Immunotherapy : Molecular Understanding of PD-1 / PD-L1 Pathway \& Translational Blockade of Immune Checkpoints. Int. J. Mol. Sci., 17, 1-22.

Yuzhakova, D., Zagaynova, E. V, Novgorod, N., Medical, S., Square, P., Novgorod, N., ... Federation, R. (2016). Immunotherapy of Cancer ( review ). CTM J, 8(1), 173-181. Zaridze, D. G. (2008). Molecular Epidemiology of Cancer. Biochem, 73(5), 532-542. 